REVISTA de LA Escuela de CIENCIAS de LA EdUCACIÓN, AÑo 15, NRO. 14, VOL. 1, ENERO A JUNIO de 2019. PÁgINAS 79-88. ISSN 1851-6297 (DESDE DICIEMBRE DE 2006 A DICIEMBRE DE 2017). ISSN 2362-3349 (EN LINEA). EXPERIENCIAS DE BUENAS PRACTICAS EN LA EDUCACIÓN SUPERIOR MATANCERA SOBRE LA BASE DE LA IGUALDAD DE GÉNERO Y EL RESPETO A LA DIVERSIDAD EN POS DE LA TRANSFORMACIÓN SOCIAL. MARIELA GONZÁLEZ ROBAINA · MABEL DOMINNGUEZ VILLALONGA · MADELIN RODRIGUEZ BENITEZ.

\title{
EXPERIENCIAS DE BUENAS PRÁCTICAS EN LA EDUCACIÓN SUPERIOR MATANCERA, SOBRE LA BASE DE LA IGUALDAD DE GÉNERO Y EL RESPETO A LA DIVERSIDAD EN POS DE LA TRANSFORMACIÓN SOCIAL
}

\author{
Mariela González Robaina* \\ Universidad de Matanzas Sede "Camilo Cienfuegos", Cuba \\ mariela.gonzalez@umcc.cu \\ Mabel Domínguez Villalonga**. \\ Universidad de Matanzas Sede "Camilo Cienfuegos", Cuba \\ mabel.dominguez@umcc.cu \\ Madelín Rodríguez Benítez \\ Universidad de Matanzas Sede "Camilo Cienfuegos", Cuba**** \\ madelìn rodrìguez@umcc.cu
}

Recibido: 29/10/2018 Aceptado: 11/12/2018

\section{Resumen}

El resultado investigativo que se presenta tiene como objetivo fundamentar la importancia del trabajo que desarrolla actualmente, el proyecto "Eco Universidad con equidad de género, y que responde a la política de la Educación Superior Cubana en pos de la transformación social. La investigación forma parte de la estrategia de la Cátedra de Género, Cultura y Sociedad" de la Universidad de Matanzas, Cuba.

En el desarrollo de la investigación se expone un análisis sintetizado del proyecto, las diferentes líneas de trabajo en las que se ha estado trabajando y se declaran los principales resultados obtenidos hasta la fecha, y que han sido valorados de manera positiva por parte de la sociedad matancera.

El trabajo está organizado por ejes temáticos que responden a las referidas líneas de trabajo, en el mismo se exponen las conclusiones y se declara la bibliografía consultada.

En el proceso investigativo se trabajó con el método investigación acción participativa, evidenciando la contribución del referido proyecto a una mejor formación humanista en las/los participantes, y al desarrollo de acciones importantes para el logro de una mayor equidad de género y el respeto a la diversidad entre las/los miembros de la comunidad intra y extra universitaria, por parte de la mencionada cátedra.

Palabras clave: Educación Superior - Género - Equidad de género - Transformación social Formación humanista.

\footnotetext{
Abstract

The research result presented aims to: Base the importance of the work currently being developed, the project "Eco University with gender equity, and which responds to the policy of the Cuban Higher Education in pursuit of social transformation. The research is part of the strategy of the Chair of Gender, Culture and Society. "Of the University of Matanzas", Cuba.

* . Máster en Ciencias de la Educación Superior. Mención Docencia Universitaria e investigación Educativa

** . Máster en Ciencias de la Educación Superior. Mención Docencia Universitaria e investigación Educativa

*** .Máster en Ciencias de la Educación Superior. Mención Docencia Universitaria e investigación Educativa
} 
REVISTA de LA ESCUELA DE CIENCIAS DE LA EDUCACIÓN, AÑo 15, NRO. 14, VOL. 1, ENERO A JUNIO dE 2019. PÁGINAS 79-88. ISSN 1851-6297 (DESDE DICIEMBRE DE 2006 A DICIEMBRE DE 2017). ISSN 2362-3349 (EN LINNEA). EXPERIENCIAS DE BUENAS PRÁCTICAS EN LA EDUCACIÓN SUPERIOR MATANCERA, SOBRE LA BASE DE LA IGUALDAD DE GÉNERO Y EL RESPETO A LA DIVERSIDAD EN POS DE LA TRANSFORMACIÓN SOCIAL. MARIELA GONZÁLEZ ROBAINA · MABEL DOMÍNGUEZ VILLALONGA · MADELIN RODRIGUEZ BENITEZ.

In the development of the research, a synthesized analysis of the project is presented, the different lines of work in which it has been working and the main results obtained to date, and which have been valued positively by societyMatancera.

The work is organized by thematic axes that respond to the aforementioned lines of work, in which the conclusions are exposed and the bibliography consulted is declared.

In the research process, we worked with the participatory action research method, evidencing the contribution of the aforementioned project to a better humanistic formation in the participants, and to the development of important actions to achieve greater gender equity and respect for the diversity among the members of the intra and extra-university community, on behalf of the aforementioned chair.

Keywords: Highereducation - Gender - Genderequity - Social transformation - Humanistic education.

\section{Introducción}

En el mundo actual y muy especialmente en nuestro país se dan muchísimos cambios y transformaciones sociales que han hecho que surjan nuevos desafíos y contradicciones para hombres y mujeres.

A partir de los acuerdos del VI Congreso y la Primera Conferencia Nacional del Partido, el proceso de fortalecimiento de la institucionalidad desarrollado en el país y la necesidad de hacer corresponder la Constitución con nuestra realidad, se elabora el proyecto de Constitución de la República aprobado por la Asamblea Nacional del Poder Popular, en su sesión ordinaria los días 21 y 22 de julio del presente año. En la etapa actual, el referido documento, se encuentra sometido a un proceso de consulta, análisis y debate popular.

Es en este proceso de transformaciones que la Educación Superior, en Cuba, deberá asumir el reto de luchar por un crecimiento pleno de las potencialidades personales y sociales, por un desarrollo sostenible, por la paz, por la equidad y la justicia social, motivo por el cual el tratamiento del tema género en el ámbito universitario y a partir de los tres procesos sustantivos, la docencia, la investigación y la extensión universitaria constituye, en el presente, el objetivo fundamental de las Cátedras de la Mujer constituidas en todas las universidades cubanas.

La capacitación y superación de la comunidad universitaria en general, en los conceptos de igualdad de género, así como de las oportunidades y posibilidades entre hombres y mujeres, contribuirá a la eliminación de estereotipos y prejuicios sexistas y a promover la equidad de género en las relaciones interpersonales. Definitivamente esta es una vía importante en que podremos superar las contradicciones y elevar la formación humanista de los/las estudiantes, y de las/los trabajadores en general.

Para poder potenciar una interpretación efectiva, en la Universidad de Matanzas, se ha diseñado un proyecto de capacitación con enfoque de género, considerando que la mayoría de las/los trabajadores y estudiantes desconocen en profundidad el tema, y cómo insertar esta problemática en sus respectivas disciplinas y asignaturas.

Aunque la implementación del proyecto muestra resultados positivos, se considera que el profesorado aún no está lo suficientemente preparado para enfrentar este reto. Múltiples son las manifestaciones de estereotipos que persisten en la sociedad cubana, a pesar de los innegables avances en cuestiones de equidad de género y particularmente las proyecciones y resultados en la ardua tarea de emancipación de la mujer, a partir de 1959.

La génesis del proyecto se establece a partir de un grupo de acciones desarrolladas por la Cátedra de Género, Cultura y Sociedad, de la Universidad de Matanzas. Las experiencias obtenidas y la incorporación en nuevos espacios de la comunidad intra y extra universitaria han servido para continuar el fortalecimiento del trabajo de capacitación en género.

A partir del diagnóstico y los talleres de reflexión realizados, desde el 2013, se han podido determinar los aspectos siguientes:

- La formación académica es importante, pero se deben propiciar mayor número de acciones que generen cambios positivos en la comunidad intra y extra universitaria.

- $\quad$ El respeto y la equidad de género, son aspectos medulares para el desarrollo social.

- El cambio se genera a partir de la propia transformación de los individuos, fomentando valores más acorde a nuestra sociedad, con un marcado carácter humanista.

- La educación cubana actual, exige individuos capaces de luchar por un mundo sostenible.

El análisis de los aspectos anteriores posibilita que la Cátedra de Género, Cultura y Sociedad, elabore y ejecute el proyecto "Eco Universidad con equidad de género".

El artículo que se presenta pretende fundamentar la importancia del trabajo que desarrolla actualmente, el proyecto "Eco Universidad con equidad de género", en la comunidad intra y extra universitaria en pos de la transformación social.

El proceso investigativo se inicia y desarrolla asumiendo el método: investigación acción participativa, con la finalidad de actuar sobre una realidad pedagógica y social existente, que permitiera la introducción 
REVISTA DE LA ESCUela dE CIENCIAS DE LA EDUCACIÓN, AÑo 15, NRO. 14, VOL. 1, ENERO A JUNIO dE 2019. PÁGINAS 79-88. ISSN 1851-6297 (DESDE DICIEMBRE DE 2006 A DICIEMBRE DE 2017). ISSN 2362-3349 (EN LINNEA). EXPERIENCIAS DE BUENAS PRÁCTICAS EN LA EDUCACIÓN SUPERIOR MATANCERA, SOBRE LA BASE DE LA IGUALDAD DE GÉNERO Y EL RESPETO A LA DIVERSIDAD EN POS DE LA TRANSFORMACIÓN SOCIAL. MARIELA GONZÁLEZ ROBAINA - MABEL DOMINGGUEZ VILLALONGA · MADELIN RODRIGUEZ BENITEZ.

de modificaciones necesarias en la práctica educativa universitaria, utilizando formas nuevas y experimentales y dando respuesta a una solicitud del Ministerio de Educación Superior unido a instituciones como El Centro de Estudios de la Mujer (CEM) y la Federación de Mujeres Cubanas (FMC)

El desarrollo de la investigación ha logrado la interacción y capacitación de las/los participantes, siendo estos los protagonistas y beneficiados del proceso. Un resultado importante ha sido el enriquecimiento del curriculum universitario con programas de capacitación en género, aplicable a las diferentes modalidades de estudio y en el desarrollo de una política educativa acorde a los requerimientos sociales en la actualidad

En el desarrollo investigativo se estableció como estrategia metodológica para la obtención de información el empleo de diferentes técnicas:

- Se determinó la confección de un diario de observación, considerando que en el mismo se han podido recoger aspectos específicos de cada actividad ejecutada, particularizando en los resultados obtenidos.

- Se realizaron grabaciones y tomaron fotografías de actividades extracurriculares, reuniones y acciones de capacitación realizadas representando importantes evidencias y referentes a la hora de diseñar acciones que contribuyeran a transformar la práctica educativa vigente.

- Se han realizado entrevistas grupales que han permitido constatar los niveles de aceptación por parte de la comunidad intra y extrauniversitaria, acerca de los estudios de género y su importancia para el desarrollo social.

Una fortaleza en el proceso de ejecución del proyecto y que ha sido un apoyo importante en la obtención de los resultados que se presentan es el nivel de compromiso y sensibilidad del equipo docente de la Cátedra de Género de la Universidad de Matanzas, en la estructuración de una estrategia de capacitación en género que tributara en la elaboración de un proyecto de intervención comunitaria en aras de establecer acciones generadoras de cambios importantes en torno a prejuicios y modos de actuación acerca de los estudios de género.

Es importante destacar que los resultados que se obtienen tributan al rigor con que se trabajaron los diferentes momentos bajo la observación detallada y reflexión, así como la coherencia en la ejecución de acciones proyectadas y ejecutadas.

Las fuentes bibliográficas consultadas han sido referentes teóricos de importancia, por los aportes y tratamiento a los estudios de género.

Finalmente, se considera que el resultado que se presenta es pertinente, en cuanto pretende socializar experiencias y resultados obtenidos que responden a necesidades reales de la Educación Superior en Cuba, así como en otros países latinoamericanos.

\section{Desarrollo \\ La universidad cubana por la equidad de género "cambiar cambiando"}

Es conocido que diferentes espacios importantes, de repercusión internacional se han proyectado en estrategias básicas para fomentar la igualdad de género, irradiando en países como Cuba. En este sentido resulta importante referirnos a:

- $\quad$ La Cuarta Conferencia Mundial sobre la Mujer, efectuada en Beijing, 1995.

- la Organización de Naciones Unidas (ONU) en aras de estructurar una línea de trabajo en los diferentes estados, se proyectó en acciones concretas.

- La Declaración y el Programa de Acción originados en la Cumbre Mundial sobre Desarrollo Social, celebrada en marzo de 1995 en Copenhague, Dinamarca.

- El Gobierno de Cuba, en abril de 1997, expresó al PNUD/UNOPS el interés de participar en la estrategia de cooperación internacional, apoyada en esos momentos por el Gobierno de Italia.

- En septiembre de 1998 quedó aprobado el documento del Programa de Desarrollo Humano a Nivel Local (PDHL-Cuba).

La Federación de Mujeres Cubanas (FMC) desde su creación el 23 de agosto de 1960, hasta la actualidad, ha dirigido y organizado el trabajo en importantes acciones que han beneficiado a la mujer cubana. Entre ellas se encuentran:

- Programa de Cátedras de la mujer en Cuba, 1989

- $\quad$ Creación del Centro de Estudios de la Mujer (CEM) de la propia FMC, 1997.

La ardua lucha por el bienestar y superación de la mujer encaminado por la FMC, así como la certera proyección de la ONU en torno a incidir en el pleno desarrollo de la mujer, unido a la misión de la universidad moderna, que, vista desde su aceptación más general, es la de preservar, desarrollar y promover, la cultura de la humanidad, a través de sus procesos sustantivos y en estrecho vínculo con la sociedad, permitieron que se diera paso a la creación de cátedras especializadas en las Universidades 
REVISTA de LA ESCUELA DE CIENCIAS DE LA EDUCACIÓN, AÑo 15, NRO. 14, VOL. 1, ENERO A JUNIO dE 2019. PÁGINAS 79-88. ISSN 1851-6297 (DESDE DICIEMBRE DE 2006 A DICIEMBRE DE 2017). ISSN 2362-3349 (EN LINNEA). EXPERIENCIAS DE BUENAS PRÁCTICAS EN LA EDUCACIÓN SUPERIOR MATANCERA, SOBRE LA BASE DE LA IGUALDAD DE GÉNERO Y EL RESPETO A LA DIVERSIDAD EN POS DE LA TRANSFORMACIÓN SOCIAL. MARIELA GONZÁLEZ ROBAINA · MABEL DOMÍNGUEZ VILLALONGA · MADELIN RODRIGUEZ BENITEZ.

Cubanas, apreciándose con ello la conveniencia de transversalizar con el enfoque de género la docencia en la enseñanza superior.

La Cátedra de "Género, Cultura y Sociedad", de la Universidad de Matanzas, se creó el 6 de Marzo de 2004, y como una propuesta realizada por la Federación de Mujeres Cubanas, y el Centro de Estudios de la Mujer, en aras de contribuir al empoderamiento de la mujer y el logro de la transformación de miembros de la comunidad intra y extra universitaria, a partir de capacitación en género. Su actividad se desarrolla en 5 áreas fundamentales:

1. Desarrollo de investigaciones.

2. Prestación de servicios a través de la documentación de la cátedra.

3. Ejecución del Proyecto extensionista: "Eco universidad con equidad de género".

4. Desarrollo de acciones de capacitación en género en la comunidad intra y extra universitaria.

5. Intercambio y socialización de resultados en diferentes eventos.

La cátedra ha desarrollado una importante labor de capacitación en diferentes espacios intra y extra universitarios. Ejecuta y promueve un programa de capacitación en género a estudiantes universitarios de diferentes carreras. Ha socializado resultados investigativos en eventos provinciales, nacionales e internacionales, obteniendo criterios favorables. Ha establecido espacios de intercambio académico con especialistas nacionales e internacionales.

El perfeccionamiento actual de los programas de desarrollo en Cuba, con una perspectiva más determinante de la sostenibilidad y la equidad, permite valorar en toda su dimensión la incorporación del tema género en acciones, a partir de la docencia, investigación y extensión.

Es necesario precisar que, antes de la elaboración e implementación del proyecto por parte de la cátedra, el enfoque de género no estaba concebido en los programas de cada una de las disciplinas y asignaturas, por no estar contemplado en los planes establecidos por el Ministerio de Educación Superior en Cuba, sin embargo, uno de los mayores aportes del proyecto "Eco universidad con equidad de género", es lograr mayor inserción del tema, en los programas y planes de estudio de cada una de las carreras.

\section{“Eco-universidad con equidad degénero". Experiencias y retos}

A partir del diagnóstico del funcionamiento y efectividad del trabajo metodológico y orientador de la cátedra, se pudieron evaluar las fortalezas y debilidades de la misma. El estudio de fuentes bibliográficas, así como el análisis de las particularidades y necesidades de las posibles áreas beneficiadas, permitió consolidar la estructura del proyecto hacia la capacitación de mujeres y hombres, en la equidad y el respeto a la diversidad para una transformación social.

Múltiples fueron los conceptos analizados, no obstante se determinó que la formación de egresados con un nivel elevado, no solo en la cultura de su profesión, sino en valores sólidos y acordes a las exigencias de la sociedad cubana actual sería la estructura rectora y el camino a seguir.

Por lo anteriormente expuesto, el ejecutivo y miembros de la Cátedra determinaron oficializar la creación del proyecto "Eco Universidad con equidad de género".

A continuación se exponen los principales objetivos que rigen la esencia del proyecto que se presenta.

Objetivo Principal

_ Contribuir a la transformación social de miembros de la comunidad intra y extra universitaria, en base a una mayor equidad de género.

Objetivos específicos

Desarrollar una visión más humanista en las/los participantes del proyecto que irradie valores positivos en miembros de la comunidad intra y extra universitaria, para lograr perfeccionar las distintas líneas de trabajo.

Estimular el debate y reflexión acerca de temas referentes a la equidad de género, a la prevención de las infecciones de transmisión sexual (ITS), al consumo de drogas, violencia entre otros, con lo cual se profundiza en las concepciones básicas.

Fortalecer la capacitación de género como vía para la transformación social, mediante promotores comunitarios.

Establecer relaciones nacionales e internacionales con instituciones y redes que trabajen la temática de género para lograr mayor profundidad científica.

Las experiencias y principales resultados obtenidos en la ejecución del proyecto han permitido concientizar la actualidad e importancia de los estudios de género.

Varias son las líneas de trabajo que conforman el proyecto: 
REVISTA de LA Escuela de CIENCIAS de LA EdUCACIÓN, AÑo 15, NRO. 14, VOL. 1, ENERO A JUNIO dE 2019. PÁGINAS 79-88. ISSN 1851-6297 (DESDE DICIEMBRE DE 2006 A DICIEMBRE DE 2017). ISSN 2362-3349 (EN LINEA). EXPERIENCIAS DE BUENAS PRÁCTICAS EN LA EDUCACIÓN SUPERIOR MATANCERA, SOBRE LA BASE DE LA IGUALDAD DE GÉNERO Y EL RESPETO A LA DIVERSIDAD EN POS DE LA TRANSFORMACIÓN SOCIAL. MARIELA GONZÁLEZ ROBAINA - MABEL DOMINGGUEZ VILLALONGA · MADELIN RODRIGUEZ BENITEZ.

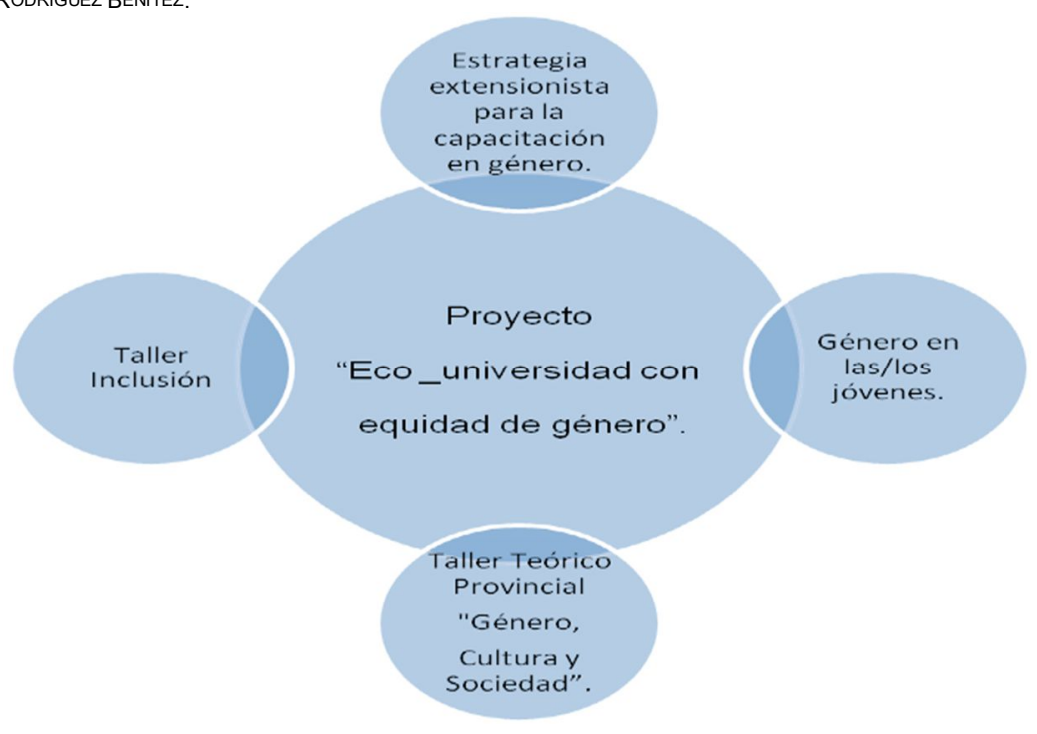

Estrategia extensionista, para la capacitación en género, de la comunidad intra y extrauniversitaria en Matanzas.

\section{“La educación no cambia el mundo, cambia a las personas que van a cambiar el mundo" Paulo Freire}

La inserción del enfoque de género en el sistema curricular universitario con una perspectiva más determinante hacia la sostenibilidad y la equidad, permite valorar en toda su dimensión la incorporación del tema, en acciones que conduzcan a una mejor calidad de las relaciones entre mujeres y hombres.

El tema género es de actualidad y su importancia para la sociedad cubana, radica en lograr un desarrollo sostenible basado en el respeto y una cultura de paz, en este sentido es importante que los egresados universitarios sea portadores de una mejor formación académica y humanista, capaces de continuar el perfeccionamiento de la obra social.

Teniendo en consideración lo expuesto anteriormente, y conscientes de que la Extensión Universitaria garantiza la estrecha relación universidad- sociedad, se valora la inclusión y fortalecimiento del tema género a partir del desarrollo de acciones de capacitación, con impacto en la comunidad intra y extrauniversitaria. El proyecto se encuentra, actualmente, en fase de implementación de una estrategia extensionista para la capacitación en género, conformada por un sistema de acciones por etapas, en cuya estructura se exponen los objetivos, contenidos, métodos, medios, formas de organización, y participantes, etc. por cada acción. También incluye acciones de retroalimentación de los resultados, así como la evaluación de la propia estrategia. La misma está dirigida a trabajadores/ras, estudiantes y miembros de la comunidad cercana a la universidad, y está organizada en tres etapas: Etapa \# 1 Diagnóstica, Etapa \# 2 Desarrollo, Etapa \# 3 Evaluación.

Principales temas trabajados:

- El Sexismo en el lenguaje.

- Roles y desempeño de las mujeres en la Historia de Cuba.

- Violencia. Actualidad y consecuencias.

- Sexualidad en mujeres y hombres.

- Drogas. Daños para la salud.

- Familia y relaciones de género.

- $\quad$ El proceso de construcción de género.

- Género en productos culturales.

Especial interés se ha venido mostrando a la impartición de un curso de postgrado, la elaboración del mismo se efectúa en el 2016, siendo aprobado por la Comisión de Postgrado de la Facultad de Ciencias Sociales y Humanidades y el Departamento Marxismo Leninismo, con fecha oficial el 14 de enero de 2015.

En el dictamen elaborado y aprobado con indicación 1/2016 de la Vicerrectoría de Investigación y Postgrado de la Universidad de Matanzas, con vigencia de 5 años, desde el 2016 hasta 2021, se hace énfasis en la correcta elaboración y adecuado grado de cientificidad de los objetivos propuestos en el curso, así como el por qué y para quienes va dirigido el curso.

Inicialmente el curso se imparte a docentes y especialistas en diferentes espacios de la provincia de Matanzas, estructurando dos grupos, en los municipios de Jovellanos y Matanzas.

En el curso 2017-2018 se procede a la impartición del curso a docentes de la Escuela Primaria: "Sí por 
REVISTA de LA ESCUELA DE CIENCIAS DE LA EDUCACIÓN, AÑo 15, NRO. 14, VOL. 1, ENERO A JUNIO dE 2019. PÁGINAS 79-88. ISSN 1851-6297 (DESDE DICIEMBRE DE 2006 A DICIEMBRE DE 2017). ISSN 2362-3349 (EN LINNEA). EXPERIENCIAS DE BUENAS PRÁCTICAS EN LA EDUCACIÓN SUPERIOR MATANCERA, SOBRE LA BASE DE LA IGUALDAD DE GÉNERO Y EL RESPETO A LA DIVERSIDAD EN POS DE LA TRANSFORMACIÓN SOCIAL. MARIELA GONZÁLEZ ROBAINA - MABEL DOMÍNGUEZ VILLALONGA · MADELIN RODRIGUEZ BENITEZ.

Cuba", del Consejo Popular de Canímar, en su fase experimental con docentes de la enseñanza primaria y como oferta de la cátedra universitaria, al mencionado centro.

En el mes de enero del presente año, se inicia el curso en el centro, con matrícula de 12 trabajadores/as.

A partir de los métodos aplicados en el proceso educativo se pudieron determinar algunas fortalezas y debilidades que incidieron posteriormente en la efectividad y resultados en el desempeño del proceso de capacitación:

Fortalezas:

- Antecedentes de acciones extracurriculares desarrolladas en el centro y comunidad, por parte de la Dirección de Extensión Universitaria, hoy Departamento de Actividades extracurriculares, de la Universidad de Matanzas.

- Previo seminario de capacitación de las prácticas inclusivas en la atención a la diversidad, recibido por docentes de la enseñanza primaria del país, con vistas al perfeccionamiento del proceso docente- educativo, en el año 2016.

- La necesidad y disposición del claustro hacia la capacitación en género, como una importante vía para lograr la eficiencia en la posterior labor educativa.

- Cercanía del Consejo Popular de Canímar a la Universidad de Matanzas.

Debilidades:

- La existencia de un horario limitado por parte del claustro docente, limitándose a dos horas, con frecuencia semanal (Hora de inicio: 1:30pm a 3:30pm).

- La limitación en medios de enseñanza para la exposición digital de materiales de interés en los temas abordados.

La evaluación constante y final de la capacitación evidenció un resultado positivo a partir de las siguientes premisas:

- En acciones desarrolladas como la proyección y debate de materiales audiovisuales:

Ejemplo: el audiovisual "Cotidianamente invisible", realizado en la Escuela: "Mártires del Goicurìa", de Matanzas, por el periodista Ayose Salvador García Naranjo, a los efectos de su tesis de graduación y que propone un acercamiento al rol que desempeña la formación escolar primaria en la transmisión de estereotipos de género, se mostró como parte del trabajo en uno de los temas impartidos. El audiovisual se apoya en entrevistas a docentes de la referida escuela y es evidente la persistencia de estereotipos que son el resultado de la cotidianidad en el proceso docente del referido centro y que estructuran roles y desempeños en las/os escolares, así como criterios despectivos en torno a conductas consideradas correctas o incorrectas como parte de la formación en los infantes.

Resultado de la actividad: En general las/los cursantes del postgrado manifestaron rechazo hacia las escenas y manifestaciones verbales de las/los entrevistados, percibiendo de forma general la existencia de estereotipos de género en docentes y estudiantes y considerando que el conocimiento recibido les brindaba las herramientas necesarias para una mejor comprensión del asunto e incidir posteriormente en cambios de pensamiento.

Algunos aspectos concluyentes en la impartición de los temas, en general:

- El grupo de cursantes del postgrado, consideró que los temas abordados les estaban aportando la posibilidad de asumir criterios guiados hacia una mejor percepción en cuanto a los estudios de género.

- Consideraron que en el desempeño escolar cotidiano existían manifestaciones de prejuicios basados en estereotipos de género tanto por parte de la familia como docentes del centro.

- La mayoría coincide en la importancia del curso como catalizador de fenómenos y actos violentos, evidenciándose específicamente en la violencia psicológica a partir del empleo de frases, tono de voz y gestualidad violenta, fruto del desconocimiento anterior sobre los tipos de violencia existentes.

- Falta de métodos para lograr la efectividad en el trabajo educativo, refieren "...no recibir ayuda de otros factores para dar solución".

- Deficiencias en el funcionamiento de las familias como eslabón fundamental en la formación de niñas/os y el estrecho vínculo que debe existir con la escuela.

- Existencia de la violencia económica, en algunos casos y que es generada en el ámbito familiar, de la que los niños/as son víctimas, manifestándose en su comportamiento y relaciones interpersonales.

- Importancia de los productos culturales consumidos a nivel social y familiar y que son reflejo de los prejuicios de 
REVISTA de LA Escuela de CIENCIAS de LA EdUCACIÓN, AÑo 15, NRO. 14, VOL. 1, ENERO A JUNIO de 2019. PÁgINAS 79-88. ISSN 1851-6297 (DESDE DICIEMBRE DE 2006 A DICIEMBRE DE 2017). ISSN 2362-3349 (EN LINEA). EXPERIENCIAS DE BUENAS PRÁCTICAS EN LA EDUCACIÓN SUPERIOR MATANCERA, SOBRE LA BASE DE LA IGUALDAD DE GÉNERO Y EL RESPETO A LA DIVERSIDAD EN POS DE LA TRANSFORMACIÓN SOCIAL. MARIELA GONZÁLEZ ROBAINA · MABEL DOMÍNGUEZ VILLALONGA · MADELIN RODRIGUEZ BENITEZ.

género que aun persisten en la sociedad cubana, visualizando la escuela como transformadora a partir de propuestas generadoras de mensajes positivos.

Propuestas de las/los cursantes, a partir del conocimiento recibido en el curso de postgrado:

- Elaborar una estrategia de trabajo en vínculo con la Universidad de Matanzas, con la finalidad de continuar desarrollando acciones con vistas al perfeccionamiento del trabajo docente educativo.

Participación del claustro en un taller de capacitación para la elaboración de productos educativos artesanales que contribuyan a ejecutar medios de enseñanza de interés para las/los infantes. En esta actividad se concibe la participación de familiares que representan un apoyo constante a la escuela y será apoyada por el Proyecto: Perfeccionamiento de la gestión Universitaria, realizándose en el mes de julio de 2018.

- Retomar la importancia del diagnóstico psicopedagógico como vía imprescindible para la adecuada atención educativa a las/los estudiantes.

- Continuar profundizando en temas referentes a las prácticas educativas inclusivas, considerando la diversidad en los centros docentes y la importancia de una adecuada atención a las diferencias individuales de las/los educandos.

- Establecer espacios de interacción con la familia y en los que se puede dar respuesta a necesidades de la comunidad, partiendo de la estimulación hacia estilos sanos de recreación e intercambio en espacios familiares y sociales.

- Promover el respeto a la diversidad, propiciando la importancia de lograr la equidad de género en la sociedad que desarrollamos, siendo una premisa en el accionar del sistema político y gubernamental cubano.

- Considerar las fortalezas y deficiencias en lo curricular, así como las posibilidades que este brinda a partir de su análisis y la elaboración de propuestas que lo potencien.

En general los principales resultados con la implementación de la estrategia muestran acciones importantes, entre las que se encuentran:

- Capacitación a cuadros de la Universidad de Matanzas, realizada en reuniones y consejos de dirección con el objetivo de capacitar a directivos y prepararlos para las diferentes etapas de implementación de la estrategia.

- Impartición del programa de capacitación en género a estudiantes de la universidad e instituciones vinculadas al proyecto. El programa abarca temas de género cuyo conocimiento es necesario como parte de la obtención de una cultura general integral en los egresados universitarios.

- Capacitación a jóvenes adolescentes de nivel secundario y pre universitario, realizada en secundarias básicas y pre universitarios del municipio Matanzas. La enseñanza en estos niveles permite seleccionar los temas que resultan interesantes para las/los jóvenes y que posibilitan establecer espacios de debate con el objetivo de incidir en reflexiones y posibles cambios en los modos de actuación juvenil.

- Diplomado de Extensión Universitaria con la inserción de un módulo sobre género. La capacitación a profesores/as representa una etapa importante de la implementación de la estrategia por cuanto las/los docentes se apropian de conocimientos sólidos que les permite obtener una visión más actualizada del tema género y las posibilidades de establecer nuevos métodos de trabajo con los estudiantes.

- Conferencias, talleres y cursos de verano. A partir de solicitudes de instituciones, el diagnóstico que se realizó en la comunidad intra y extra universitaria y sobre la base del criterio de expertos se ha ofertado la capacitación considerando las particularidades de cada modalidad.

- Elaboración e impartición de cursos de postgrado de Capacitación en género, dirigido a docentes y especialistas de la provincia, con el propósito de lograr ampliar espacios de superación en el tema.

\section{Taller Inclusión}

Por la importancia del tema género, las/los miembros de la cátedra valoran la idea de promover acciones y programas educativos que fomenten el conocimiento sobre temas de género, sexualidad, envejecimiento con calidad, familia y medio ambiente, facilitando el desarrollo de actitudes, valores y conocimientos en todas las edades y poblaciones con vistas a lograr total plenitud de hombres y mujeres.

Como respuesta a las preocupaciones e intereses planteados anteriormente se crean los talleres: "Inclusión", diseñados y estructurados para realizarse en grupos poblacionales con particularidades diferentes, el aprendizaje de técnicas artesanales y el debate de temas de género 
REVISTA de LA ESCUELA DE CIENCIAS DE LA EDUCACIÓN, AÑo 15, NRO. 14, VOL. 1, ENERO A JUNIO dE 2019. PÁGINAS 79-88. ISSN 1851-6297 (DESDE DICIEMBRE DE 2006 A DICIEMBRE DE 2017). ISSN 2362-3349 (EN LINNEA). EXPERIENCIAS DE BUENAS PRÁCTICAS EN LA EDUCACIÓN SUPERIOR MATANCERA, SOBRE LA BASE DE LA IGUALDAD DE GÉNERO Y EL RESPETO A LA DIVERSIDAD EN POS DE LA TRANSFORMACIÓN SOCIAL. MARIELA GONZÁLEZ ROBAINA · MABEL DOMÍNGUEZ VILLALONGA · MADELIN RODRIGUEZ BENITEZ.

para las/los participantes resultó la premisa rectora en cada encuentro. Entre los beneficiados están:

- Instituciones culturales y proyectos comunitarios de la ciudad de Matanzas.

- Mujeres de la tercera edad del Centro Cultural "Nelson Barrera" de la Comunidad "La Marina".

- Grupo heterogéneo de mujeres, convocadas a recibir los talleres del proyecto universitario y que están siendo apoyadas por el Programa de Personas Mayores de Caritas Matanzas, radicando en el Consejo Popular de Pueblo Nuevo.

- $\quad$ Niños/as, en diferentes espacios de la ciudad matancera.

Objetivo general:

- Contribuir a través de las manualidades y el debate de temas de género, a mejorar la calidad de vida de las/los participantes.

Los temas seleccionados responden a los intereses de la Cátedra, así como a las particularidades de las/los participantes, contando con la participación de expertos de diferentes organismos.

En el caso de las adultas mayores representó una experiencia importante ya que se trabajó bajo la visión de ampliar su calidad de vida además de servirles como beneficio económico y espiritual. Los grupos de adultas mayores fueron motivados a su vez, para convertirse en promotoras de la Cátedra, en la comunidad y apoyar a las profesoras con el trabajo con otros grupos, en la propia comunidad, escuelas primarias y secundarias o empresas que lo solicitaran.

\section{Género en las/los jóvenes}

El proyecto ha desarrollado acciones de capacitación en escuelas de nivel secundario y pre universitario de la provincia de Matanzas.

Objetivo general.

_ Demostrar la importancia de los estudios de los problemas de género en la adolescencia.

Principales acciones:

- Talleres sobre diferentes temas de interés en la adolescencia: Tatuaje y piercing. Mitos y realidades, violencia. Actualidad y consecuencias, socialización de género. Mundo azul-mundo rosado, roles de género y la familia en la sociedad actual.

- Actividades de intercambio cultural entre las/los jóvenes, con la finalidad de desarrollarlas a partir de los intereses de las/los implicados.

Resultados obtenidos:

_ Trabajo comunitario en espacios del Consejo Popular de Pueblo Nuevo, Matanzas:

- Casa Cáritas: Talleres de creación con niñas/os y adolescentes, así como espacios de reflexión en torno a temas de actualidad y que contribuyen a la formación de valores.

- Callejón de las tradiciones: Trabajo en la impartición de talleres de intercambio sobre temas de género u otros de interés para la comunidad y que contribuyan a mejorar las relaciones interpersonales, así como establecer espacios de recreación sana y de disfrute en sus pobladores.

\section{Taller Teórico Provincial "Género, Cultura y Sociedad"}

El trabajo desarrollado por la cátedra hasta la fecha, el interés asumido por la comunidad universitaria en el desarrollo de investigaciones que respondieran específicamente a temas referentes a los estudios de género, así como la necesidad de establecer espacios de socialización que propicien un intercambio académico importante sobre el tema dan lugar a la organización del primer Taller Teórico Provincial de "Género, Cultura y Sociedad", en el año 2006.

El objetivo general del taller, en sus diferentes ediciones, ha sido: Motivar la investigación y desarrollo hacia estudios feministas y estudios de género, contribuyendo a la difusión y socialización de los principales resultados investigativos de profesores/as, investigadores/as y expertos en general.

Cada uno de los Talleres Teóricos Provinciales, realizados con carácter anual, han permitido determinar el grado o comportamiento de las relaciones de género en los diferentes espacios universitarios, empresariales y sociales.

Entre los temas de mayor interés, abordados en los encuentros, se pueden mencionar el impacto de proyectos comunitarios en las relaciones interpersonales, el sexismo en el lenguaje, sexualidad en la tercera edad, comportamiento de la violencia en la actualidad, género y acceso de poder en las empresas, género desde el proceso docente educativo, entre otros.

La ejecución del taller se logra a partir de un diseño estructurado en temáticas específicas que dan respuesta al objetivo propuesto en cada edición:

- La mujer cubana y su rol en el desarrollo integral de la sociedad. 
REVISTA DE LA ESCUela dE CIENCIAS DE LA EDUCACIÓN, AÑo 15, NRO. 14, VOL. 1, ENERO A JUNIO dE 2019. PÁGINAS 79-88. ISSN 1851-6297 (DESDE DICIEMBRE DE 2006 A DICIEMBRE DE 2017). ISSN 2362-3349 (EN LINEA). EXPERIENCIAS DE BUENAS PRÁCTICAS EN LA EDUCACIÓN SUPERIOR MATANCERA, SOBRE LA BASE DE LA IGUALDAD DE GÉNERO Y EL RESPETO A LA DIVERSIDAD EN POS DE LA TRANSFORMACIÓN SOCIAL. MARIELA GONZÁLEZ ROBAINA - MABEL DOMINNGUEZ VILLALONGA - MADELIN RODRIGUEZ BENITEZ.

- Evaluación del impacto de la capacitación de género a partir de la implementación de estrategias, planes y proyectos universitarios.

En el resultado que presenta la ejecución de los talleres, se puede evidenciar el nivel de excelencia alcanzado progresivamente en cada uno, la participación e implicación de diferentes especialistas y personas interesadas del ámbito social y académico matancero.

En general es importante referir que los resultados obtenidos, como parte del proyecto, han sido socializados en los eventos siguientes:

_Taller Nacional de Empoderamiento en Adultas Mayores. Cienfuegos, enero 2018.

- Taller Nacional sobre experiencias del trabajo comunitario con adultas mayores sobre la base de la equidad de género. Varadero, enero 2018. $201 \overline{8}$

Encuentro de educadores cubanos y norteamericanos. Asociación de Pedagogos de Cuba. Febrero

Participación en Universidad 2018.

Evento CIUM, 2018

_Taller Provincial de Género, Universidad de Matanzas. Marzo 2018.

\section{Conclusiones}

A modo de conclusión se puede afirmar la factibilidad del proyecto ya que la cátedra ha logrado incentivar a las/los profesores, trabajadores y estudiantes con acciones docentes e investigativas intra y extra universitarias. Se ha logrado mayor inserción en espacios intra y extra universitarios, las acciones desarrolladas con la tercera edad ha implicado un estímulo relevante para los ancianos de casas de abuelos, por manifestar interés en rescatar habilidades perdidas y conocer nuevas, el trabajo desarrollado en las comunidades, ha tenido como acción fundamental, el estudio de los diferentes tipos de violencia ejercida hacia la mujer.

Por lo expuesto anteriormente se propone dar continuidad y enriquecer las acciones siguientes:

- Establecer mayor inserción de los estudios de género en el desarrollo formativo de cada facultad que integra la universidad de Matanzas, tributando en lograr que el $100 \%$ del estudiantado universitario complete su formación con dominio y sensibilidad en los temas afines a los mencionados estudios.

- Dar continuidad a la impartición del postgrado de capacitación en género, considerando irradiar en diversos espacios de la sociedad.

- Continuar desarrollando acciones que contribuyan al empoderamiento de las mujeres y mayor bienestar y calidad de vida en la vejez.

- Continuar la búsqueda y asesoramiento a intereses investigativos en el ámbito social matancero, generando resultados que permitan transformaciones necesarias en la sociedad actual.

\section{Referencias bibliográficas}

- $\quad$ Álvarez Suárez, M. (2014). Familia y Género. Continuidad y rupturas. Editorial de la Mujer, Cuba.

- Calviño, M. (2017). Descubriendo la psicología. Editorial Academia, Cuba.

- $\quad$ Colectivo de autores. (2012) .Acercamiento a la teoría de género. CEM, Cuba.

- $\quad$ (2009). Camino a la igualdad de género. UNIFEM, Cuba. - (2004).Capacitación en género y Desarrollo Humano. Editorial Científico -Técnica, Cuba. Editorial de la Mujer, Cuba. (2008).Cátedras de la Mujer en Cuba: Experiencias de transversalización de género. (2012). La discriminación de género en el derecho y sus expresiones en la - legislación y en la práctica jurídica. CEM, Cuba.

- (2016). Mujeres en desarrollo. Guía metodológica para la formación de competencias info-comunicativas para el emprendimiento femenino. Editorial Universitaria, Cuba. (1993). Mujeres Latinoamericanas en cifras: Cuba. Instituto de la Mujer, Cuba.

- $\quad$ (s/f) Ni con el pétalo de una rosa, Violencia de género. Editorial de la Mujer, Cuba. - $\quad(s / f)$ Orientaciones metodológicas para el trabajo de las Cátedras de la Mujer. Centro de Estudio de la Mujer, Cuba.

- $\quad$ (2007). Pasajes de género en Cuba. CEM, Cuba.

(2007). Pasajes de género en Cuba. CEM, Cuba.
(2013). Rompiendo silencios: Lecturas sobre mujeres, géneros y desarrollo humano. Editorial de la mujer, Cuba.

(s/f) Subjetividad de género en Cuba: Las construcciones socioculturales de los femenino y lo masculino. Perspectivas de cambio. CEM, Cuba.

(2011). Violencia de género en las familias, encrucijadas para el cambio, Centro de Investigaciones Psicológicas y Sociológicas. CEM, Cuba.

- $\quad$ Fleitas Ruiz, R. y Ávila Vargas, N. G. (2013).Salud y Sexualidad. CEM, Cuba

- Fleitas Ruiz, R. y Romero Almodóvar, M. (2012). Familia, género y violencia doméstica. Instituto Cubano de Investigación Cultural Juan Marinello, Cuba.

- González Hernández, A., B; Castellanos S. y cols, (1994). "Estereotipos sexuales: masculinidad y feminidad en la edad escolar". Revista Educación No. 82/mayo-agosto, Cuba.

- González Pagés, J. C. (2010). Macho Varón Masculino: Estudios de masculinidades en Cuba. Editorial de la Mujer, Cuba.

- González Robaina, M. (2010).experiencias en la transversalización de género, a :Cátedra de la mujer 
REVISTA de LA ESCUELA DE CIENCIAS DE LA EDUCACIÓN, AÑo 15, NRO. 14, VOL. 1, ENERO A JUNIO dE 2019. PÁGINAS 79-88. ISSN 1851-6297 (DESDE DICIEMBRE DE 2006 A DICIEMBRE DE 2017). ISSN 2362-3349 (EN LINNEA). EXPERIENCIAS DE BUENAS PRÁCTICAS EN LA EDUCACIÓN SUPERIOR MATANCERA, SOBRE LA BASE DE LA IGUALDAD DE GÉNERO Y EL RESPETO A LA DIVERSIDAD EN POS DE LA TRANSFORMACIÓN SOCIAL. MARIELA GONZÁLEZ ROBAINA - MABEL DOMÍNGUEZ VILLALONGA · MADELIN RODRIGUEZ BENITEZ.

partir de la extensión universitaria. Congreso Internacional Universidad 2010, Cuba.

- Leyva Fuentes, M. (2016). La labor preventiva desde el mejoramiento de las pràcticas inclusivas en la atención a la diversidad. Editorial Pueblo y Educación, Cuba.

- $\quad$ Licea Díaz, O. (2006). Ecología Interior. Editorial Científico Técnica, Cuba.

- Proveyer Cervantes, C. (2005).Selección de lecturas de Sociología y Política Social de Género. Editorial Félix Varela, Cuba.

- Sainz de Robles, F. C. (2012). Diccionario Español de sinónimos y antónimos. Editorial José Martí, Cuba.

- Velázquez Sánchez, L. M. (2012). Carrera y desarrollo profesional de la mujer. Editorial Libio, Cuba. 